\title{
Short-circuiting long-lived humoral immunity by the heightened engagement of CD40
}

\author{
Loren D. Erickson, ${ }^{1}$ Brigit G. Durell, ${ }^{1}$ Laura A. Vogel, ${ }^{2}$ Brian P. O’Connor, ${ }^{1}$ \\ Marilia Cascalho, ${ }^{3}$ Teruhito Yasui, ${ }^{4}$ Hitoshi Kikutani, ${ }^{4}$ and Randolph J. Noelle ${ }^{1}$ \\ ${ }^{1}$ Department of Microbiology, Dartmouth Medical School, Lebanon, New Hampshire, USA \\ ${ }^{2}$ Department of Biological Sciences, Illinois State University, Normal, Illinois, USA \\ ${ }^{3}$ Department of Surgery and Immunology, Mayo Medical School, Rochester, Minnesota, USA \\ ${ }^{4}$ Department of Molecular Immunology, Research Institute for Microbial Diseases, Osaka University, Osaka, Japan \\ Address correspondence to: Randolph J. Noelle, Department of Microbiology, Dartmouth Medical School, \\ 1 Medical Center Drive, Lebanon, New Hampshire 03756, USA. \\ Phone: (603) 650-7670; Fax: (603) 650-6223; E-mail: rjn@dartmouth.edu.
}

Received for publication August 30, 2001, and accepted in revised form January 21, 2002.

Agonistic $\alpha \mathrm{CD} 40 \mathrm{Ab}$ 's have been shown to be potent immune adjuvants for both cell- and humoralmediated immunity. While enhancing short-lived humoral immunity, the administration of a CD40 agonist during thymus-dependent immune responses ablates germinal center formation, prematurely terminates the humoral immune response, blocks the generation of $\mathrm{B}$ cell memory, and prevents the generation of long-lived bone marrow plasma cells. Interestingly, some of these effects of heightened CD40 engagement could be mimicked by enhancing the magnitude of antigen-specific $\mathrm{T}$ cell help. Taken together, these studies demonstrate that as the magnitude of CD40 signaling intensifies, the fate of antigen-reactive $B$ cells can be dramatically altered. These are the first studies to describe the multifaceted function of CD40 in determining the fate of antigen-reactive $B$ cells and provide novel insights into how CD40 agonists can short-circuit humoral immunity.

J. Clin. Invest. 109:613-620 (2002). DOI:10.1172/JCI200214110.

\section{Introduction}

Interrupting CD154-CD40 interactions prevents the development of thymus-dependent (TD) humoral responses and many cell-mediated immune responses (1). Conversely, it has been shown that agonistic $\alpha C D 40 \mathrm{Ab}$ 's administered in vivo can act as potent immune adjuvants for both arms of the immune response. Several reports have shown that $\alpha \mathrm{CD} 40$ enhances the immune response to thymus-independent (TI) antigens $(2,3)$ and can amplify cell-mediated immune responses to viruses and tumors (4-6). Given these observations, the clinical use of CD40 agonists to boost the immune response of the immunocompromised host has been considered.

We have investigated whether CD40 agonists can influence the follicular versus extrafollicular differentiation of antigen-experienced B cells. Antigen-specific B cells that are activated by CD154-dependent cognate interactions with $\mathrm{T}$ cells traffic either to follicles or to extrafollicular foci. Follicular B cells in the spleen that are recruited to form germinal centers (GCs) ultimately differentiate to memory B cells and/or to long-lived plasma cells that migrate to the bone marrow where they sustain high-affinity, antigen-specific Ig levels in the serum (7-11). In contrast, B cells that emigrate to extrafollicular foci within the red pulp, following interactions with $\mathrm{T}$ cells, locally differentiate to short-lived Ab-secreting cells (ASCs) that produce low-affinity Ig $(12-14)$. Here, we document that the administration of a CD40 agonist ( $\alpha \mathrm{CD} 40 \mathrm{mAb})$, in combination with a TD antigen, ablates GC formation and induces a pattern of extrafollicular B cell differentiation in the spleen and lymph node. This CD40-induced deviation resulted in a heightened early humoral immune response, the premature termination of the humoral immunity, loss of long-lived bone marrow plasma cells, and memory B cell development. Interestingly, priming of the T cell compartment to enhance CD40 signaling mimicked some but not all of the effects of the $\alpha \mathrm{CD} 40$ agonist. This latter data indicates that extrafollicular B cell differentiation by heightened CD40 signaling may represent a physiological means to limit the duration and intensity of the humoral immune response. Taken together, these studies demonstrate that $\alpha \mathrm{CD} 40$, as well as the magnitude of helper $T$ cell activity, can influence the follicular/extrafollicular fate of antigen-reactive B cells.

\section{Methods}

Mice. C57/BL6 Ly5.2 congenic mice and CD40-/C57/BL6 mice, 6-8 weeks old, were bred and maintained in the specific pathogen-free animal facility at Dartmouth Medical School, Lebanon, New Hampshire, USA. QM mice were backbred to the C57/BL6 $\mathrm{JH}^{-/-} \mathrm{Jk}_{\mathrm{k}}{ }^{-/-}$ strain for nine generations to produce $\mathrm{QM} \mathrm{F}_{9}$ C57/BL6 mice. IghCD40 mice were generated as described previously (15). Briefly, wild-type human CD40 cDNA was cloned into the $\mathrm{pEmVhp}$ expression vector containing the mouse rearranged $\mathrm{H}$ chain promoter derived from 
pMO- $\mu 4 \mathrm{C} 8$ and microinjected into C57/BL6 oocytes. Mice were backcrossed to murine $\mathrm{CD}^{-1 /-}$ mice of a C57/BL6 background, thereby allowing the expression of human CD40 exclusively in the B cell compartment. $B$ cell preparation and adoptive transfer. Spleen cell suspensions isolated from QM mice were treated with ammonium chloride-Tris buffer and were $T$ cell depleted using anti-Thy-1.2 plus anti-CD4 Ab and rabbit complement (Accurate Chemical \& Scientific Corp., Westbury, New York, USA). An aliquot of cells was stained with the R2348.8 4-hydroxy-5-nitrophenylacetyl (NP) idiotype-specific $\mathrm{mAb}$ and the pan $\mathrm{B}$ cell marker, B220, and analyzed by flow cytometry (FACScan; Becton Dickinson Immunocytometry Systems, San Diego, California, USA) to determine percentage of $\mathrm{Tg} B$ cells. In all experiments, normal rat serum was added to minimize nonspecific staining. Tg B cells $\left(3 \times 10^{6}\right)$ were adoptively transferred intravenously into sex-matched C57/BL6 Ly5.2 recipient mice. All experiments consisted of three mice per group.

Immunization and $\alpha \mathrm{CD} 40$ treatment. $\mathrm{NP}_{30}-\mathrm{KLH}$ and NPFicoll were purchased from Solid Phase Sciences Inc. (San Rafael, California, USA). To examine TD responses, recipient mice were challenged intraperitoneally 24 hours after adoptive transfer with CFA alone or with $100 \mu \mathrm{g}$ NP-KLH emulsified in CFA in the absence or presence of FGK115. FGK115, an agonistic rat $\operatorname{IgG}_{2 a}$ anti-mouse CD40, was HPLC-purified and administered at $100 \mu \mathrm{g}$ intraperitoneally on day 0,2 , and 4 after immunization. As indicated, control rat IgG was administered similarly to FGK115. In some experiments, mice were immunized intraperitoneally with $200 \mu \mathrm{l}$ of a $10 \%$ solution of sheep blood alsevers (Colorado Serum Co., Denver, Colorado, USA) and treated with S2C6 (16), an agonistic anti-human CD40 mAb. Mice were killed on day 7 , and intact spleens were removed and sera collected by retro-orbital eye bleeds. For experiments using carrier-primed $\mathrm{T}$ cells, recipient mice were challenged intraperitoneally with $100 \mu \mathrm{g}$ KLH emulsified in CFA 1 week prior to cell transfer. Recipients were immunized intraperitoneally and subcutaneously with $100 \mu \mathrm{g}$ NP$\mathrm{KLH}$ precipitated in alum, and analyses were performed on day 5 after immunization. Secondary immune responses were assessed by pooling $\mathrm{T}$ cell-depleted splenic B cells from primary recipients 30 days after immunization and by transferring $10 \times 10^{6}$ cells intravenously into secondary recipients that were KLHprimed 2 weeks previously. Hosts were challenged with alum alone or with $100 \mu \mathrm{g}$ NP-KLH alum 24 hours after transfer, and serum was collected 7 days later. Endogenous levels of high-affinity anti-NP IgG $\mathrm{Ig}_{1}$ and $\mathrm{IgG}_{2 \mathrm{a}}$ were detected by ELISA. Long-lived bone marrow ASCs generated from the spleen or lymph node were quantified by ELISPOT 7 days after transferring normalized numbers of $\mathrm{Tg} B$ cells into naive secondary hosts.

Flow cytometric reagents. The following $\mathrm{mAbs}$ were used for multicolor flow-cytometric analysis: 6B2, a rat $\mathrm{IgG}_{2 \mathrm{a}}$; anti-mouse B220, a rat IgM anti-mouse GL7; and CD45.2, a rat $\mathrm{IgG}_{2 \mathrm{a}}$ anti-mouse Ly5.1 and Ly5.2, were purchased from PharMingen (San Diego, California, USA). FITC-conjugated $\mathrm{Ab} 90$, a rat $\operatorname{IgG}_{2 a}$ anti-mouse CD38, and FITC-conjugated peanut agglutinin (PNA) were purchased from Sigma Chemical Co. (St. Louis, Missouri, USA). The anti- $\mathrm{V}_{\mathrm{H}}$ 17.2.25 cell line (R2348.8) was generously provided by Theresa Imanishi-Kari (University of California San Francisco, San Francisco, California, USA). Streptavidin-phycoerythrin (streptavidin-PE) and FITC were obtained from Southern Biotechnologies Associates (Birmingham, Alabama, USA). For removal of cytophilic Ig, cells were washed in $5 \%$ FCS in BSS and resuspended in low-pH ( $\mathrm{pH} 4.0)$ acetate buffer containing $0.05 \mathrm{M} \mathrm{Na}$ acetate, $0.085 \mathrm{M}$ $\mathrm{NaCl}$, and $0.005 \mathrm{M} \mathrm{KCl}$ and $2 \% \mathrm{FCS}$ in distilled $\mathrm{H}_{2} \mathrm{O}$. Samples were incubated on ice for 1 minute followed by the addition of an equal volume of $0.1 \mathrm{M}$ Tris buffer, $\mathrm{pH} 8.0$, containing $2 \%$ FCS. Samples were washed twice in 5\% FCS BSS and then stained. Chromatographically purified rat $\operatorname{IgG}$ was used as isotype control. A minimum of 200,000 events per sample were collected on a FACScan, and dead cells were excluded based on forward and $90^{\circ}$ light scatter.

ELISA and ELISPOT reagents. NP-25-BSA or NP-4-BSA was used to capture NP-specific Ab's in a sandwichbased ELISA. Allotypic-specific (Igh ${ }^{\mathrm{a}}$ or Igh ${ }^{\mathrm{b}}$ ) goat antimouse $\operatorname{IgG}_{1}$ and $\operatorname{IgG}_{2 \mathrm{a}}$ conjugated to alkaline phosphatase (Southern Biotechnology Associates) were used to detect $A b$ derived from $T g$ or endogenous $B$ cells with subsequent development with phosphatase substrate (Sigma Chemical Co.). Absorbance was read at a dual wavelength of 405 and $540 \mathrm{~nm}$ using an MR700 plate reader (Dynatech Laboratories, Chantilly, Virginia, USA). Serum from a hyperimmunized QM mouse was used as a standard in all assays to determine nanograms per milliliter. For ELISPOT analysis, a modified manufacturer's protocol was used from Millipore Corp. (Beverly, Massachusetts, USA). Briefly, Millipore MultiScreen HA plates were coated with either NP-25-BSA or NP-4-BSA. Normalized numbers of Tg B cells were plated in triplicate at a starting concentration of $2.4 \times 10^{6}$ cells, serially titrated, and incubated at $37^{\circ} \mathrm{C}$ for 5 hours. $\mathrm{Tg}$ ASCs were detected with allotype-specific $\left(\operatorname{Igh}^{\mathrm{a}}\right)$ biotin-conjugated rat anti-mouse IgG1a followed by development with streptavidin-horse radish peroxidase and chromogen substrate, and counted.

Confocal image analysis. On day 5 or 7 after immunization, intact spleens and lymph nodes were harvested and 4 - to $5-\mu \mathrm{m}$ frozen sections were fixed in acetone at $4^{\circ} \mathrm{C}$ for 15 minutes and allowed to air dry at $4^{\circ} \mathrm{C}$ for 48 hours prior to staining. Sections were stained in $1 \%$ BSA PBS using a three-color protocol with rat antimouse Ab's: 6B2 FITC, CD4-, and CD8-PE, and Cy5conjugated anti-NP idiotype $\mathrm{V}_{\mathrm{H}}$ 17.2.25. To block nonspecific binding, $1 \%$ mouse serum and $10 \%$ normal rat serum were included in the staining process. Samples were analyzed on a Bio-Rad 1024 confocal microscope (Bio-Rad Laboratories Inc., Hercules, California, USA). RT-PCR. On day 7 after immunization, T cell-depleted splenocytes were dual stained with biotin-conjugat- 
ed anti-NP idiotype $V_{\mathrm{H}}$ 17.2.25 and Ly5.1 to label Tg B cells. Tg B cells were positively selected ( $\geq 90 \%$ purity) using streptavidin-coupled microbeads (Miltenyi Biotec, Auburn, California, USA). Total RNA ( $1 \mu$ g each) isolated from purified Tg B cells by using RNeasy system (QIAGEN Inc., Valencia, California, USA) was used as a template for cDNA synthesis with Superscript II System (Life Technologies Inc., Rockville, Maryland, USA). PCR amplification was carried out by titrating cDNA samples with Taq (Boehringer Mannheim $\mathrm{GmbH}$, Mannheim, Germany) and the primers for HPRT to normalize levels of template. Amplification of BSAP and Blimp-1 transcripts were carried out using normalized amounts of cDNA. The primers used were as follows: 5'-GCTGGTGAAAAGGACCTCT-3' (HPRT forward) and $5^{\prime}$-CACAGGACTAGAACACCTGC-3' (HPRT reverse); $5^{\prime}$-TCCTCGGACCATCAGGACAG-3' (BSAP forward) and $5^{\prime}$-CCTGTTGATGGAGCTGACGC-3' (BSAP reverse); $5^{\prime}$-TCCGGCTCCGTGAAGTTTCAA-3' (Blimp-1 forward) and 5'-GGTGGAACTCCTCTCTGGAAT-3' (Blimp-1 reverse). Conditions used for amplification were $94^{\circ} \mathrm{C}$, $55^{\circ} \mathrm{C} / 65^{\circ} \mathrm{C}$, and $72^{\circ} \mathrm{C}$ for 35 cycles. $H P R T$ was used as a control to confirm the amount of each template.

\section{Results}

Anti- $(\alpha) C D 40$ administration aborts GC B cell differentiation. We have developed a system using Tg B cells that faithfully recapitulates the humoral immune response to TD antigens and provides the means to track clonal
B cell behavior in vivo. Tg B cells can be clearly identified by virtue of their antigen specificity to NP, the expression of the $\mathrm{V}_{\mathrm{H}}$ 17.2.25 idiotype, and by the expression of the allotypic marker, Ly5.1, when using congenic Ly5.2 mice as recipients. Tg B cells vigorously expand by day 7 after challenge with the TD antigen, NP-KLH (2). To evaluate how CD40 agonists alter clonal expansion and differentiation of $\mathrm{B}$ cells during TD responses, recipients were immunized in the presence or absence of agonistic $\alpha \mathrm{CD} 40 \mathrm{mAb}$. Total numbers of $\mathrm{Tg} B$ cells present in the spleens of immune mice were quantified at day 7 after immunization. Immune $\mathrm{Tg} B$ cells expanded approximately threefold over naive control animals, regardless of control Ig or $\alpha \mathrm{CD} 40$ treatment (Figure 1a). As demonstrated here and reported previously (2), $\operatorname{Tg} B$ cells failed to undergo expansion in recipients that were administered $\alpha \mathrm{CD} 40$ in the absence of antigen. These results indicate that $\mathrm{Tg} B$ cell expansion is antigen-driven and that increasing CD40 engagement by the addition of $\alpha \mathrm{CD} 40 \mathrm{mAb}$ does not enhance the proliferative phase of $\mathrm{Tg} B$ cell activation. A hallmark of follicular B cell differentiation is the generation of GCs. GC formation and kinetics in this Tg system can be readily quantified by flow cytometric analysis of the $\mathrm{Tg} B$ cells using a panel of GC-specific markers (17-19). $\mathrm{Tg} B$ cells from the spleens of immune recipients exhibited a GC phenotype as demonstrated by the upregulation of PNA and GL7 staining and the downregulation of CD38 expression (Figure 1b). In con-
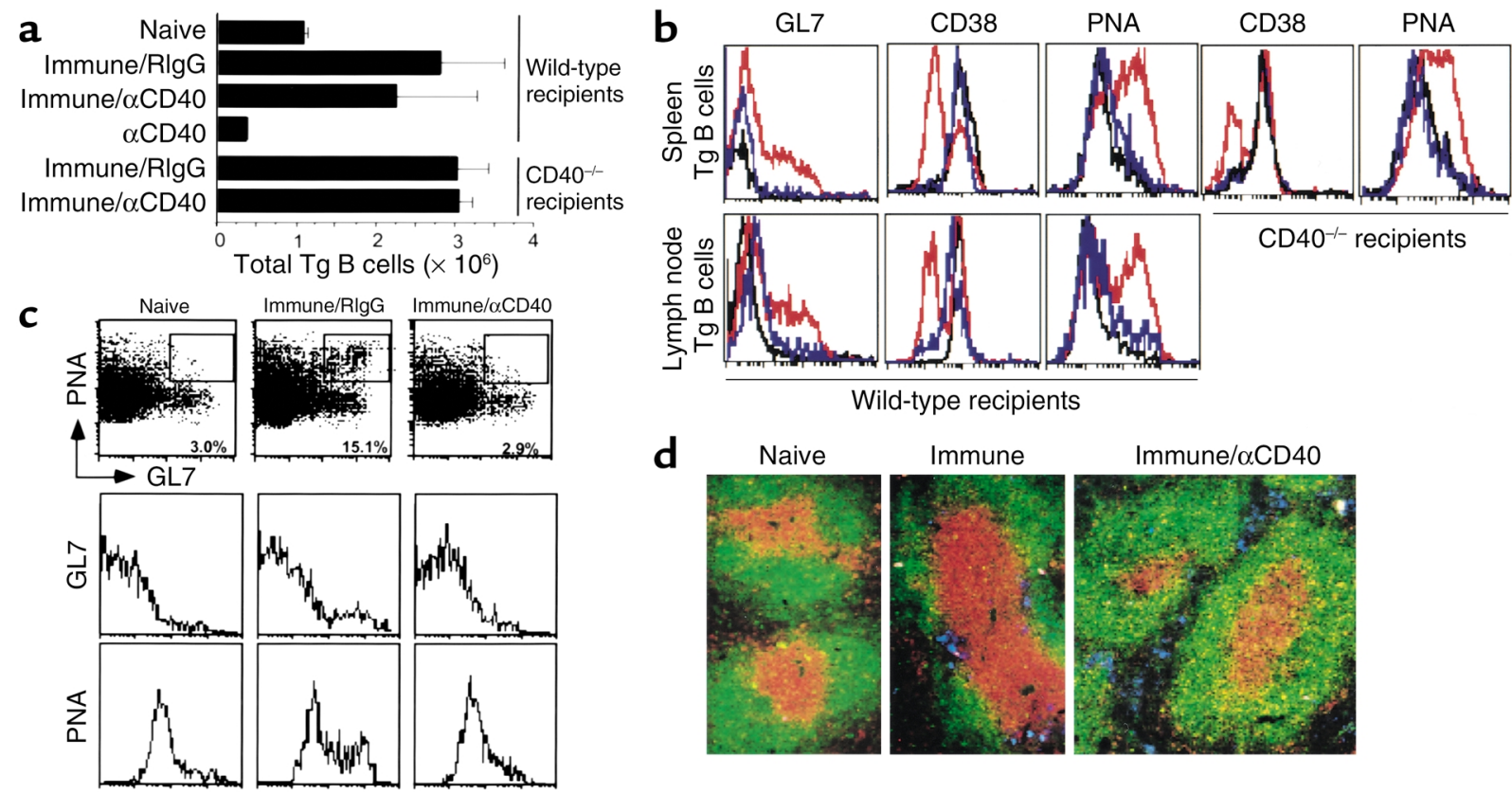

Figure 1

$\alpha C D 40$ treatment aborts a GC phenotype and promotes extrafollicular localization of Tg B cells. (a) Total numbers of splenic Tg B cells (anti$\mathrm{NP}$ idiotype ${ }^{+}, \mathrm{B} 220^{+}$) were measured on day 7 after immunization. (b) Spleen or LN cells from naive (black line), immune/RlgG (red line), and immune $/ \alpha C D 40$ (blue line) were stained with GC markers. Histograms shown represent the relative intensity of the indicated marker on $\mathrm{Tg} B$ cells $\left(B 220^{+} \mathrm{Id}^{+}\right)$. (c) Spleen cells from naive and immune IghCD40 transgenic mice treated with rat IgG or agonistic $\alpha \mathrm{hCD} 40 \mathrm{mAb}$ were stained with GC markers. The percentage of total PNA ${ }^{+} G L 7^{+} \mathrm{GC} B$ cells are indicated in each panel. (d) Frozen sections from intact spleens were costained with B220 (green), CD4, and CD8 (red), and anti-NP idiotype (Id) 17.2.25 (blue) Ab's, and analyzed by confocal microscopy. Objective: $\times 20$; zoom 1.5. Data are representative of three independent experiments. 


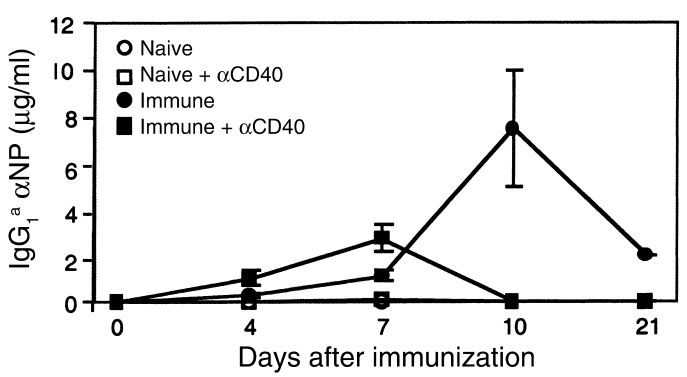

Figure 2

Administration of $\alpha C D 40$ reduces the duration of humoral immune responses. Serum $\alpha N P \operatorname{lgG}{ }_{1}$ a levels were measured by ELISA on days indicated after immunization. Values are a composite of three mice per group. Data are representative of three experiments.

trast, $\mathrm{Tg} B$ cells from immune recipients treated with $\alpha C D 40$ lacked all three markers of GC phenotype, yet expanded similar to mice challenged with antigen alone. $\mathrm{Tg} \mathrm{B}$ cells isolated from lymph nodes (LNs) of immune recipients expanded, and, as observed in the spleen, treatment with $\alpha \mathrm{CD} 40$ prevented the emergence of a GC phenotype (Figure 1b). Extensive analysis of Tg B cells at time points earlier or later (from days 2-9) than day 7 (from LNs or spleen) demonstrated that $\alpha \mathrm{CD} 40$ administration universally prevented the appearance of a GC phenotype. Hence, the failure of immune $\mathrm{Tg} B$ cells to acquire a GC phenotype with $\alpha \mathrm{CD} 40$ treatment was not due to a delay or an early onset in the kinetics of GC formation (data not shown).

To determine whether the loss in GC phenotype with $\alpha \mathrm{CD} 40$ treatment was a direct effect on Tg B cells, adoptive transfer experiments were carried out in $\mathrm{CD} 40^{-/-}$ recipients. Similar to findings in wild-type recipients, $\mathrm{Tg}$ $\mathrm{B}$ cells in immune $\mathrm{CD} 40^{-/-}$recipients showed a threefold expansion over naive controls, regardless of $\alpha \mathrm{CD} 40$ administration (Figure 1a). Results also showed that $\alpha C D 40$ administration prevents the acquisition of a GC phenotype on immune $\mathrm{Tg} \mathrm{B}$ cells in $\mathrm{CD} 40^{-/-}$mice (Figure $1 \mathrm{~b})$. These findings strongly suggest that $\alpha \mathrm{CD} 40$ administration is exerting its effect directly on $\mathrm{Tg} B$ cells and not via an indirect effect on other CD40-bearing accessory cells. Thus, CD40 signaling provided by endogenous helper $T$ cells induced expansion and follicular B cell differentiation in immune recipients, and $\alpha \mathrm{CD} 40$ treatment significantly diverted the capacity of these cells to acquire a GC phenotype and enter the follicular differentiation pathway.

In an effort to further confirm the in vivo effects of $\alpha \mathrm{CD} 40$ treatment on B cell differentiation, we performed similar experiments using transgenic mice (IghCD40) that express human CD40 exclusively in the B cell compartment. IghCD40 mice were immunized with sheep red blood cells in the presence or absence of the agonistic anti-human CD40 mAb, S2C6 (16). B cells from the spleens of immune mice exhibited a GC phenotype as shown by PNA and GL7 expression (Figure 1c). In contrast, immune mice treated with S2C6 prevented the acquisition of GC B cells. These findings indicate that the differential effect of exaggerated CD40 signaling on $\mathrm{B}$ cell responses is not unique to a particular TD antigen or agonistic $\alpha \mathrm{CD} 40 \mathrm{mAb}$. Moreover, these results provide additional evidence that $\alpha \mathrm{CD} 40$ treatment acts directly on antigen-activated $B$ cells.

We examined the histology of spleen sections from recipient mice to determine the anatomical localization of Tg B cells. On day 7 after immunization (peak expansion), tissue sections taken from intact spleens of naive or immune mice without or treated with $\alpha \mathrm{CD} 40$ were stained with B220, CD4, and CD8, and anti-NP idiotype $V_{H}$ 17.2.25 to delineate $\mathrm{Tg} B$ cells within the $B$ and $T$ cell areas. Results showed that immunization with a TD antigen, in the absence of $\alpha \mathrm{CD} 40$, induced the appearance of several clusters of immune $\mathrm{Tg} B$ cells $\left(\mathrm{B} 220^{+} \mathrm{Id}^{+}\right)$located at the interface between the $\mathrm{T}$ cell-rich zone of the periarteriolar lymphoid sheath and the surrounding follicular B cells (Figure 1d). In contrast, $\mathrm{Tg} B$ cells from immune recipients treated with $\alpha \mathrm{CD} 40$ were found exclusively in the extrafollicular space of the red pulp. These findings are consistent with the lack of GC phenotype and demonstrate that when CD40 engagement is heightened during TD responses, antigen-reactive $B$ cells are diverted to the extrafollicular areas in the spleen.

CD40 agonists reduce the duration of humoral immune responses and ablate the generation of $B$ cell memory. The dramatic alterations in follicular $B$ cell differentiation induced by CD40 agonists would predict that CD40 agonists should alter the intensity or duration of the overall humoral immune response. Hence, the magnitude and longevity of the serum $\operatorname{IgG}_{1}{ }^{a}$ anti-NP (derived from $\mathrm{Tg} B$ cells) was measured over a 3-week interval when $\alpha C D 40$ was or was not administered. $\alpha \mathrm{CD} 40$ treatment of immune recipients facilitated an earlier onset of NP-specific $\operatorname{IgG}_{1}{ }^{a}$ production and showed twofold greater amounts of $\operatorname{IgG}_{1}{ }^{a}$ anti-NP over mice challenged with NP-KLH alone at day 7 after immunization (Figure 2). Furthermore, $\alpha \mathrm{CD} 40$ treatment altered the kinetics of $\operatorname{IgG}_{1}{ }^{a}$ anti-NP responses by caus-

\section{Table 1}

$\alpha C D 40$ treatment ablates secondary immune responses

\begin{tabular}{lccc} 
& Primary & \multicolumn{2}{c}{ NP-4 binding } \\
recipients & $\begin{array}{l}\text { Secondary } \\
\text { recipients }\end{array}$ & $\operatorname{IgG}_{1}{ }^{A}$ & $\operatorname{IgG}_{2 a}$ \\
Naive & naive & n.d. ${ }^{B}$ & n.d. \\
Naive & immune & n.d. & n.d. \\
Immune & naive & $0.26 \pm 0.22$ & n.d. \\
Immune & immune & $9.64 \pm 2.7$ & $32.4 \pm 3.0$ \\
Naive $+\alpha C D 40$ & naive & n.d. & n.d. \\
Naive $+\alpha C D 40$ & immune & n.d. & n.d. \\
Immune $+\alpha C D 40$ & naive & n.d. & n.d. \\
Immune $+\alpha C D 40$ & immune & $0.25 \pm 0.14$ & $0.41 \pm 0.01$
\end{tabular}

Primary recipient mice were immunized with CFA (naive) or $100 \mu \mathrm{g}$ NP-KLH CFA (immune) in the absence or presence of $\alpha$ CD 40 . After 30 days, splenic $B$ cells from each group were adoptively transferred into carrier-primed secondary recipients and challenged with alum (naive) or $100 \mu \mathrm{g} \mathrm{NP-KLH}$ alum (immune). Titers of high-affinity serum IgG levels were measured by ELISA on day 7 after immunization. Data are representative of three experiments. ${ }^{A} \mu \mathrm{g} / \mathrm{ml}$. ${ }^{B}$ Not detected $(<2.0 \mathrm{ng} / \mathrm{ml})$. 

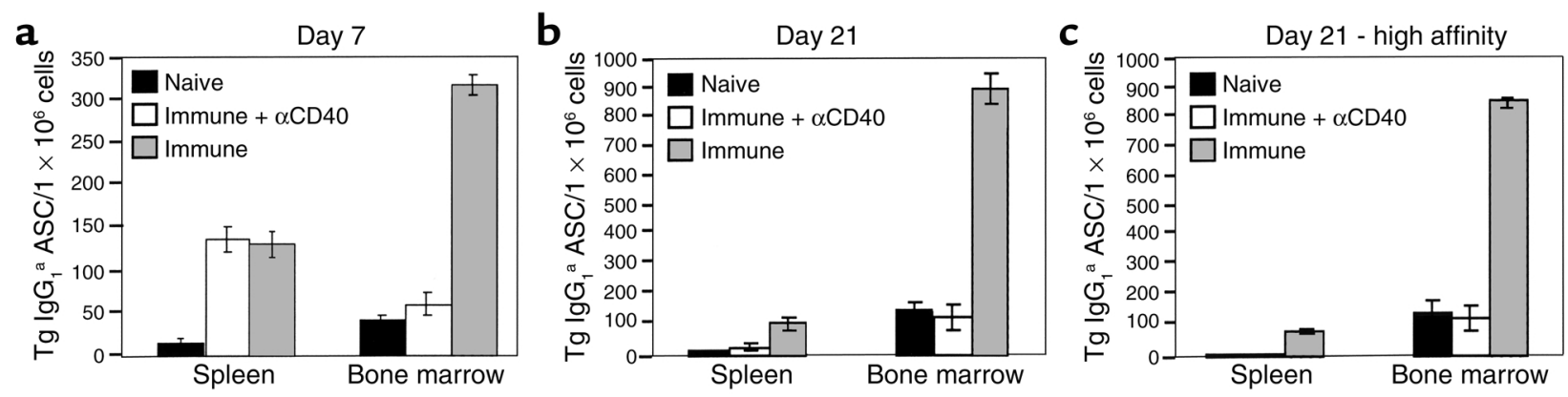

Figure 3

$\alpha C D 40$ administration prevents the generation of bone marrow ASCs. Cells from spleen and bone marrow were isolated from the indicated recipients on day 7 (a) and day 21 (b) after immunization and assayed for total NP-specific $\lg _{1} \mathrm{G}_{1}{ }$ or high-affinity NP-specific $\lg \mathrm{G}_{1}{ }^{\mathrm{a}}(\mathbf{c})$ by ELISPOT using normalized numbers of Tg B cells. Results shown are representative of three independent experiments.

ing the premature termination of responses by 10 days after immunization. Thus, $\alpha \mathrm{CD} 40$ administration modestly enhanced early humoral immune responses but terminated the humoral immune response prematurely. Since it appeared that $\alpha \mathrm{CD} 40$ aborted GC formation and prematurely terminated humoral immunity, we assessed whether $\alpha \mathrm{CD} 40$ could prevent the generation of NP-specific memory B cells using normal, non- $\mathrm{Tg} B$ cells. Mice were immunized with NP-KLH and administered $\alpha \mathrm{CD} 40$ or control rat Ig. After 30 days, B cells were isolated, transferred to secondary recipients, and challenged with antigen. Mice that received immune splenic $B$ cells produced significant titers of high-affinity NPspecific $\operatorname{IgG}_{1}{ }^{\mathrm{b}}$ and $\operatorname{IgG}_{2 \mathrm{a}} \mathrm{b}$ upon antigenic challenge (Table 1). In contrast, secondary hosts that were given $B$ cells from mice that were immunized and administered $\alpha \mathrm{CD} 40$ failed to generate a memory response when rechallenged with antigen. Thus, $\alpha \mathrm{CD} 40$ administration prevents the generation of memory B cells.

$\alpha C D 40$ agonists prevent the generation of long-lived plasma cells in the bone marrow. Previous reports have shown that a significant proportion of splenic Ab-secreting cells (ASCs) that develop from extrafollicular differentiation have a lifespan of less than 3 days $(13,20)$, thus providing local short-term $\mathrm{Ab}$ production. Study of the generation of long-lived plasma cells has revealed that many of these are likewise derived in the spleen, but unlike short-lived ASCs, long-lived plasma cells develop from the follicular pathway and migrate to the bone marrow where they can persist for months $(10,21)$. Because $\alpha C D 40$ treatment terminates humoral immunity prematurely, we anticipated an impact of $\alpha \mathrm{CD} 40$ treatment on the generation of long-lived plasma cells. To examine the production of short- and long-lived ASCs in our studies, we performed ELISPOT analyses of spleen and bone marrow in immune mice treated with $\alpha \mathrm{CD} 40$. Results from these studies demonstrated that $\mathrm{Tg} \mathrm{IgG}_{1}{ }^{a}$ ASCs were present in the spleen of all immune recipients 7 days after challenge (Figure 3a). Analysis of splenic ASCs generated by day 21 demonstrated a reduction in $\mathrm{Tg} \mathrm{IgG}_{1}{ }^{\mathrm{a}}$ ASCs in all recipients compared with day 7 and illustrated the short-lived nature of most of the splenic ASCs identified (Figure 3b). High numbers of long-lived
$\operatorname{IgG}_{1}{ }^{a}$ ASCs were apparent in the bone marrow of immune mice and the majority of these underwent affinity maturation to produce high-affinity (NP-4-binding) $\mathrm{Ab}$ (Figure 3c). The administration of $\alpha \mathrm{CD} 40$ caused a complete ablation of long-lived bone marrow Tg plasma cells on all days tested. These results show that the terminal differentiation of follicular B cells to long-lived plasma cells is ablated when CD40 signaling is elevated during TD immune responses and explains the shortlived nature of the humoral immune response.

CD40 agonists alter the patterns of Blimp-1 and BSAP expression. The fact that $\alpha C D 40$ caused a marked diversion in $B$ cell differentiation led us to investigate if alterations in differentiation-specific transcription factor expression could be observed. BSAP (B lymphocyte-specific activation protein) and Blimp-1 (B lymphocyte-induced maturation protein 1) are transcriptional factors that have been shown to be expressed during distinct phases of $B$ cell differentiation. $B S A P$ is expressed on all differentiating B cells, except for cells that have terminally differentiated into short-lived ASCs or plasma cells residing in the bone marrow (22). In contrast, Blimp-1 gene expression is rapidly upregulated in B cells that have terminally differentiated to ASCs and plasma cells $(23,24)$. We examined

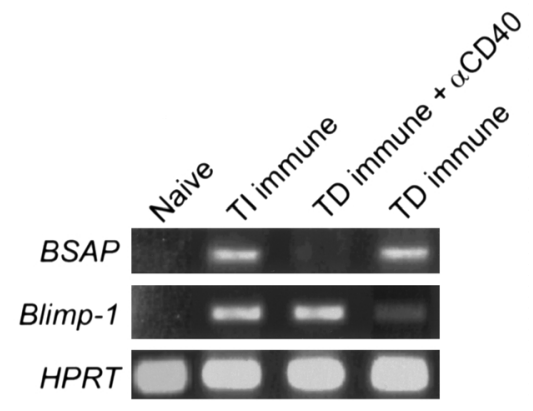

Figure 4

Administration of $\alpha$ CD40 upregulates Blimp-1 gene expression in Tg B cells. PCR amplification of the indicated transcript was performed using normalized amounts of CDNA isolated from purified splenic $\mathrm{Tg}$ B cells of recipients challenged with NP-Ficoll (TI immune), NP-KLH (TD immune), or NP-KLH and treated with $\alpha C D 40$ (TD immune $+\alpha C D 40)$. Data are representative of three mice per group from two independent experiments. 
a

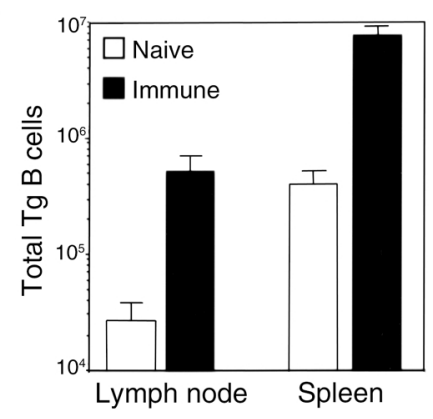

C
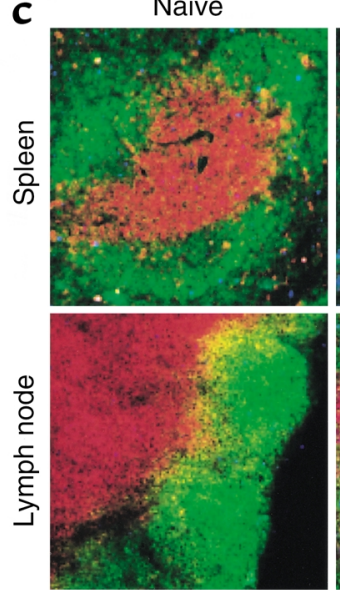

b
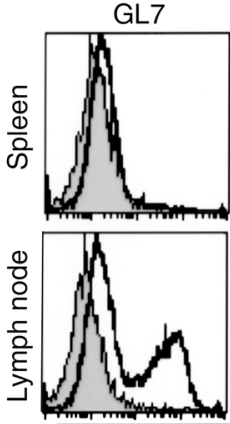

Immune

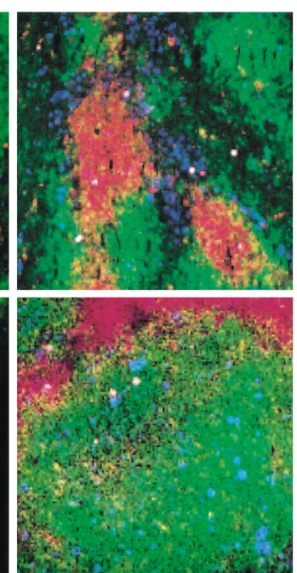

d

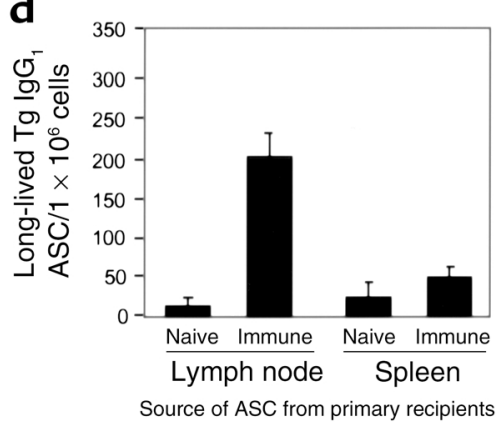

Figure 5

Carrier-primed $T$ cells prevent the acquisition of a GC phenotype in the spleen but not in the LN and differentially control the generation of long-lived Tg ASCs. (a) Total numbers of $L N$ and splenic Tg B cells were quantified 5 days after challenge. (b) Tg cells from naive (shaded area) and immune (open area) recipients were stained for the expression of the indicated GC marker. Histograms shown represent the relative intensity on gated $\mathrm{Tg}$ cells isolated from $\mathrm{LN}$ and spleen. Data are representative of six experiments. (c) Frozen sections from intact spleen and LN were costained with B220 (green), CD4 and CD8 (red), and anti-NP Id 17.2.25 (blue) Ab's, followed by confocal image analysis. Objective: $\times 20$; zoom: 1.75 . (d) Equivalent numbers of naive and immune $\mathrm{Tg} B$ cells isolated from spleen and $L N$ of carrier-primed recipients were transferred into secondary recipients that received neither priming nor antigen. Total numbers of bone marrow NP-specific $\operatorname{lgG}_{1}$ a ASCs were quantified by ELISPOT analysis 7 days after transfer. Data shown are representative of three independent experiments. whether these transcriptional factors were regulated in vivo by $\mathrm{CD} 40$ agonists during TD immune responses. $\mathrm{Tg}$ B cells from recipients were purified on day 7 to $90 \%$ or more by magnetic bead selection using the congenic marker Ly5.1, and mRNA levels of BSAP and Blimp-1 were evaluated. Adequate numbers of $\mathrm{Tg} B$ cells could be isolated only at the peak of expansion (day 7) and limited the extent of the analysis. RT-PCR analysis demonstrated that BSAP mRNA was expressed at high levels in $\mathrm{Tg} B$ cells isolated from recipients that were challenged with NP-KLH compared with naive Tg cells (Figure 4). In contrast, Blimp-1 mRNA expression was present at low levels in immune $\mathrm{Tg}$ cells. The differential expression of these transcription factors support the notion that during TD responses, $\operatorname{Tg} \mathrm{B}$ cells are actively undergoing follicular differentiation. The low levels of Blimp-1 expression from these follicular $B$ cells is not surprising since terminal differentiation of long-lived plasma cell precursors occurs in the bone marrow. Significantly, BSAP mRNA expression was very low in immune $\operatorname{Tg} B$ cells that had been purified from recipients treated with $\alpha \mathrm{CD} 40$, but high levels of Blimp-1 gene expression were observed. As an additional control, $\operatorname{Tg} \mathrm{B}$ cells were also purified from recipients that were challenged with the TI antigen, NP-Ficoll, and tested for mRNA expression levels of BSAP and Blimp-1. B cells that respond to TI antigens do not require cognate interactions with helper $\mathrm{T}$ cells for activation and therefore culminate in the early activation and local differentiation of B cells to short-lived ASCs (25). Both BSAP and Blimp-1 expression levels were elevated in TI-immune Tg
B cells, as expected. These data demonstrate that elevated levels of CD40 signaling diverts B cells along the extrafollicular differentiation pathway and differentiates locally into short-lived ASCs.

Enhanced Tcell help mimics some, but not all, of the effects of CD40 agonists on follicular B cell differentiation. The above findings suggest that enhanced CD40 engagement by $\alpha \mathrm{CD} 40$ administration directs antigen-reactive $B$ cells to the extrafollicular differentiation pathway. While this provides valuable insights into how CD40 agonists may alter the humoral immune response, does enhanced CD40 engagement by helper T cells (Th cells) exert similar immunological effects on $\mathrm{B}$ cell differentiation? The magnitude of CD40 signaling of B cells by Th cells could be enhanced by the increased frequency of Th cells and density of CD154 on memory Th cells. Priming the $T$ cell compartment with antigen in CFA would both increase the frequency of carrier-reactive Th cells and produce a population of memory $T h$ cells that have higher densities of CD154 (26).

We tested whether $\mathrm{T}$ cell priming causes alterations in $\mathrm{B}$ cell differentiation by using recipient mice that were hyperimmunized with the carrier, $\mathrm{KLH}$, in CFA. Following the transfer of $\mathrm{Tg} \mathrm{B}$ cells into the carrierprimed recipients, they were challenged (intraperitoneally and subcutaneously) with alum alone or NPKLH precipitated in alum. This systemic and regional immunization assured $\mathrm{T}$ cell priming both in the spleen and LN. In mice that were carrier-primed, immune Tg B cells in both LN and spleen expanded 
approximately tenfold over naive controls (Figure 5a). These levels of B cell expansion were consistently higher than those observed in recipients that were not KLHprimed (Figure 1a) and demonstrated that carrierprimed $\mathrm{T}$ cells provided superior helper activity to naive $T$ cells and that they were present in the spleen and LN.

The differentiation of Tg B cells in the LN and spleen from naive and carrier-primed immune recipients were evaluated by staining with the GC markers GL7, CD38, and PNA. Immune $\mathrm{Tg} B$ cells from the spleen of carrierprimed recipients profoundly expand, however, they fail to express a GC phenotype (Figure 5b), whereas in noncarrier primed mice, Tg B cells do express GC markers in the spleen (Figure 1b). In contrast to that observed in the spleen, immune $\mathrm{Tg} B$ cells taken from $\mathrm{LN}$ of identical recipients fully differentiated into follicular GC B cells $\left(\mathrm{GL7}^{+} \mathrm{CD}^{-} 8^{-} \mathrm{PNA}^{+}\right)$. That is, in an individual mouse, $\mathrm{Tg} \mathrm{B}$ cells expanded in both the $\mathrm{LN}$ and spleen, but in the spleen they diverted to an extrafollicular pathway and in the LN to a follicular pathway. These results were confirmed by examining the histology of spleen and LN sections from carrier-primed recipients. Similar to the histology of $\alpha \mathrm{CD} 40$-treated mice (Figure 1d), extrafollicular localization of splenic Tg B cells was observed (Figure 5c). In contrast, $\mathrm{Tg} B$ cells from the LNs of the same primed recipients had entered follicles.

The observation that GC formation was selectively overridden by carrier-primed Th cells in the spleen and not the LN implied that long-lived plasma cells may only be generated in the LN under these prescribed conditions. To test this hypothesis, Tg B cells from primed spleen and LN were passively transferred into recipient mice, and the number of ASCs in the bone marrow of recipient mice was determined. $\mathrm{Tg} B$ cells from primed $\mathrm{LN}$ gave rise to higher numbers of bone marrow plasma cells (Figure 5d). While we cannot directly attribute these effects to heightened CD154 expression on Th cells, the data suggest that when the immune response intensifies, the spleen assumes the responsibility for the production of short-lived, extrafollicular ASCs, while the LN continues to drive B cells through a follicular GC-type response.

\section{Discussion}

The studies presented show that the differentiation pathway of antigen-experienced B cells can be altered by a CD40 agonist or the intensity of $\mathrm{T}$ cell help. We demonstrate that the administration of a CD40 agonist induces extrafollicular $B$ cell differentiation resulting in the loss in GC formation, a modest increase in serum Ig levels, the premature termination of the humoral immune response, the ablation of long-lived bone marrow plasma cells, and the loss in the generation of $\mathrm{B}$ cell memory. The fate of antigen-reactive $\mathrm{B}$ cells can also be altered by heightened $\mathrm{T}$ cell help, however, in a more restricted, organ-specific fashion.

There has been much interest as to the role of CD40 in the terminal differentiation of $\mathrm{B}$ cells. The results presented herein lead to the conclusion that $\alpha \mathrm{CD} 40$ admin- istration in vivo directs extrafollicular B cell differentiation. However, in vitro studies of the role of CD40 agonists on the differentiation of $\mathrm{B}$ cells are conflicting. On one hand, two published reports have shown that CD40 signaling in vitro can prevent $B$ cell differentiation to plasma cells $(27,28)$. Arpin et al. have shown that human GC cells cultured with the cytokines IL-2 and IL-10 and CD154-transfected cells acquire a memory B cell phenotype and that the withdrawal of CD154 resulted in terminal differentiation to ASCs (29). On the other hand, a number of reports have shown CD40 signaling in vitro directly induces $\mathrm{B}$ cell differentiation and $\mathrm{Ab}$ secretion (30-32). First, in vivo and in vitro approaches are vastly different, and second, the in vitro studies were performed in the absence of antigen. Our own studies show that the impact of CD40 agonists are only observed in the presence of antigen and preclude $B$ cells from entering the follicular pathway.

In agreement with our data showing that heightened levels of CD 40 signaling promotes extrafollicular B cell differentiation, a recent study by Uchida et al. has demonstrated that overexpression of a constitutive CD40-like signaling molecule, LMP-1, promotes extrafollicular differentiation (33). Uchida and colleagues demonstrated that antigenic challenge of LMP- $1 \mathrm{Tg}$ mice induced extrafollicular B cell responses and failed to initiate GC formation and the production of high-affinity Ab's. As with the $\alpha \mathrm{CD} 40$ agonist, signaling through LMP-1 actively suppresses GC formation of differentiating $B$ cells and fosters extrafollicular differentiation. It is noteworthy that Epstein-Barr virus-transformed B cells detected in lymphoid tissues of infectious mononucleosis patients are found neither in primary follicles nor GCs, but in the extrafollicular areas $(34,35)$. All of these data are in accord with the hypothesis that the triggering of the CD40 signaling cascade by a CD40 agonist or expression of LMP- 1 diverts $B$ cell differentiation.

One potential mechanism for the in vivo effects of $\alpha \mathrm{CD} 40 \mathrm{mAb}$ treatment on $\mathrm{B}$ cell differentiation is that the $\mathrm{mAb}$ may interfere with $\mathrm{B}$ cell migration. Experimental data showing that GC responses were ablated in the spleens of carrier-primed recipients suggest that $\mathrm{mAb}$ treatment does not directly alter $\mathrm{B}$ cell migration. Of interest is whether the differences in GC responses between spleen and LN of hyperimmune mice are due to differences in chemokine responsiveness. A recent study by Hargreaves and coworkers demonstrated that as splenic B cells differentiate into ASCs they undergo changes in their ability to respond to chemokines (36).

Understanding the impact of CD 40 agonists on B cell differentiation in vivo both has practical advantages and provides insights into the physiological function of CD40 in terminal B cell differentiation. With regard to the former, CD40 agonists have received attention as potential adjuvants in vaccine development. The in vivo use of agonistic $\alpha \mathrm{CD} 40 \mathrm{Ab}$ 's has been shown previously to enhance isotype-switched $\mathrm{Ab}$ responses to TI antigens such as pneumococcal polysaccharides (3) and to ameliorate collagen-induced arthritis with long-term 
$\alpha \mathrm{CD} 40$ treatment (37). However, our studies demonstrate that while $\alpha \mathrm{CD} 40$ administration can transiently enhance antigen-specific Ig levels, it can also enforce the premature termination of long-lived humoral immunity and B cell memory. These findings raise issues with the use of CD40 agonists for enhancing long-term immunity. From a physiological standpoint, these studies have uncovered a novel effect of CD40 agonism during a normal immune response. In a primary immune response, antigen-specific GC responses were readily observed in both the spleen and LN. However, when the helper $\mathrm{T}$ cell compartment was hyperimmune, the response in the spleen was diverted to a nonGC, extrafollicular response, while the LN sustained a GC reaction. More in-depth analysis showed that in a carrier-primed mouse the Tg B cells from the LN, but not the spleen, could develop into bone marrow plasma cells. At this time, we cannot directly attribute the differential effects in the spleen versus $L N$ to heightened CD154 expression on the antigen-reactive Th cells, but it is consistent with data presented using a CD40 agonist. More importantly, these studies suggest that as the immune response matures and $\mathrm{T}$ cell help intensifies, the function of the spleen and LN diverge, and each assumes distinct responsibilities for sustaining the humoral immune response. Late in an immune response, we would hypothesize that the spleen acquires the responsibility of producing short-lived ASCs, while the $\mathrm{LN}$ produces memory B cells and long-lived ASCs for export to the bone marrow. A more extensive testing of this novel hypothesis is currently underway.

\section{Acknowledgments}

The authors would like to thank A.G. Rolink and S. Paulie for generously providing the FGK115 (anti-mouse CD40) and S2C6 (anti-human CD40) mAbs, respective$\mathrm{ly}$, and T. Imanishi-Kari for the kind gift of the R2348.8 cell line (anti- $\mathrm{V}_{\mathrm{H}}$ 17.2.25). The $\mathrm{C} 57 / \mathrm{BL} \mathrm{J}_{\mathrm{H}}{ }^{-/-}$and $\mathrm{Jk}_{\mathrm{k}}{ }^{-/-}$mice were kindly supplied by D. Nemazee. This work was supported by grants from the NIH to R.J. Noelle (AI-26296 and AI-42234) and L.D. Erickson (AI-10302-01A1).

1. Foy, T., Aruffo, A., Bajorath, J., Buhlmann, J.E., and Noelle, R.J. 1996. Immune regulation by CD40 and its ligand gp39. Annu. Rev. Immunol. 14:591-617.

2. Erickson, L.D., et al. 2000. B cell immunopoiesis: visualizing the impact of CD40 engagement on the course of T cell-independent immune responses in an Ig transgenic system. Eur. J. Immunol. 30:3121-3131.

3. Dullforce, P., Sutton, D.C., and Heath, A.W. 1998. Enhancement of T cellindependent immune responses in vivo by CD40 antibodies. Nat. Med. 4:88-91.

4. Schoenberger, S.P., Toes, R.E.M., van der Voort, E.I.H., Offringa, R., and Melief, C.J.M. 1998. T-cell help for cytotoxic T lymphocytes is mediated by CD40-CD40L interactions. Nature. 393:480-483.

5. Bennett, S.R.M., et al. 1998. Help for cytotoxic T-cell responses is mediated by CD40 signaling. Nature. 393:478-480.

6. French, R.R., Chan, H.T., Tutt, A.L., and Glennie, M.J. 1999. CD40 antibody evokes a cytotoxic T-cell response that eradicates lymphoma and bypasses T-cell help. Nat. Med. 5:548-553.

7. Jacob, J., and Kelsoe, G. 1992. In situ studies of the primary immune response to (4-hydroxy-3-nitrophenyl)acetyl. II. A common clonal origin for periarteriolar lymphoid sheath-associated foci and germinal centers. J. Exp. Med. 176:679-687.

8. Nossal, G.J.V. 1992. The molecular and cellular basis of affinity maturation in the antibody response. Cell. 68:1-2.
9. MacLennan, I.C. 1994. Germinal centers. Annu. Rev. Immunol. 12:117-139. 10. Slifka, M.K., Antia, R., Whitmire, J.K., and Ahmed, R. 1998. Humoral immunity due to long-lived plasma cells. Immunity. 8:363-372.

11. Ochsenbein, A.F., et al. 2000. Protective long-term antibody memory by antigen-driven and $T$ help-dependent differentiation of long-lived memory B cells to short-lived plasma cells independent of secondary lymphoid organs. Proc. Natl. Acad. Sci. USA. 97:13263-13268.

12. Jacob, J., Kassir, R., and Kelsoe, G. 1991. In situ studies of the primary immune response to (4-hydroxy-3-nitrophenyl)acetyl. I. The architecture and dynamics of responding cell populations. J. Exp. Med. 173:1165-1175.

13. Ho, F., Lortan, J.E., MacLennan, I.C., and Khan, M. 1986. Distinct shortlived and long-lived antibody-producing cell populations. Eur. J. Immunol. 16:1297-1301.

14. Sze, D.M., Toellner, K.M., de Vinuesa, C.G., Taylor, D.R., and MacLennan, I.C. 2000. Intrinsic constraint on plasmablast growth and extrinsic limits of plasma cell survival. J. Exp. Med. 192:813-822.

15. Yasui, T., et al. 2002. Dissection of B cell differentiation during primary immune responses in mice with altered CD40 signals. Int Immunol. In press.

16. Paulie, S., et al. 1984. Monoclonal antibodies to antigens associated with transitional cell carcinoma of the human urinary bladder. II. Identification of the cellular target structures by immunoprecipitation and SDSPAGE analysis. Cancer Immunol. Immunother. 17:173-179.

17. Allman, D.M., Ferguson, S.E., Lentz, V.M., and Cancro, M.P. 1993. Peripheral B cell maturation II. Heat-stable antigen(hi) splenic B cells are an immature developmental intermediate in the production of long-lived marrow-derived B cells. J. Immunol. 151:4431-4444.

18. Laszlo, G., Hathcock, K.S., Dickler, H.B., and Hodes, R.J. 1993. Characterization of a novel cell-surface molecule expressed on subpopulations of activated T and B cells. J. Immunol. 150:5252-5262.

19. Oliver, A.M., Martin, F., and Kearney, J.F. 1997. Mouse CD38 is down-regulated on germinal center B cells and mature plasma cells. J. Immunol. 158:1108-1115.

20. Smith, K.G., Hewitson, T.D., Nossal, G.J., and Tarlinton, D.M. 1996. The phenotype and fate of the antibody-forming cells of the splenic foci. Eur. J. Immunol. 26:444-448.

21. Manz, R.A., Thiel, A., and Radbruch, A. 1997. A lifetime of plasma cells in the bone marrow. Nature. 388:133-134

22. Neurath, M.F., Stuber, E.R., and Strober, W. 1995. BSAP: a key regulator of B-cell development and differentiation. Immunol. Today. 16:564-569.

23. Turner, C.A., Jr., Mack, D.H., and Davis, M.M. 1994. Blimp-1, a novel zinc finger-containing protein that can drive the maturation of B lymphocytes into immunoglobulin-secreting cells. Cell. 77:297-306.

24. Schliephake, D.E., and Schimpl, A. 1996. Blimp-1 overcomes the block in IgM secretion in lipopolysaccharide/anti-mu $\mathrm{F}\left(\mathrm{ab}^{\prime}\right) 2$-co-stimulated B lymphocytes. Eur. J. Immunol. 26:268-271.

25. Mond, J.J., Lees, A., and Snapper, C.M. 1995. T cell-independent antigens type 2. Annu. Rev. Immunol. 13:655-692.

26. Jaiswal, A.I., and Croft, M. 1997. CD40 ligand induction on T cell subsets by peptide-presenting B cells: implications for development of the primary T and B cell response. J. Immunol. 159:2282-2291.

27. Callard, R.E., Herbert, J., Smith, S.H., Armitage, R.J., and Costelloe, K.E. 1995. CD40 cross-linking inhibits specific antibody production by human B cells. Int. Immunol. 7:1809-1815.

28. Lane, P., Burdet, C., McConnell, F., Lanzavecchia, A., and Padovan, E. 1995. CD40 ligand-independent B cell activation revealed by CD40 ligand-deficient $\mathrm{T}$ cell clones: evidence for distinct activation requirements for antibody formation and B cell proliferation. Eur. J. Immunol. 25:1788-1793.

29. Arpin, C., et al. 1995. Generation of memory B cells and plasma cells in vitro. Science. 268:720-722.

30. Spriggs, M.K. et al. 1992. Recombinant human CD40 ligand stimulates B cell proliferation and immunoglobulin E secretion. J. Exp. Med. 176:1543-1550.

31. Grabstein, K.H., et al. 1993. The regulation of T cell-dependent antibody formation in vitro by CD40 ligand and IL-2. J. Immunol. 150:3141-3147.

32. Maliszewski, C.R., et al. 1993. Recombinant CD40 ligand stimulation of murine B cell growth and differentiation: cooperative effects of cytokines. Eur. J. Immunol. 23:1044-1049.

33. Uchida, J., et al. 1999. Mimicry of CD40 signals by Epstein-Barr virus LMP1 in B lymphocyte responses. Science. 286:300-303.

34. Pallesen, G., Hamilton-Dutoit, S.J., Rowe, M., and Young, L.S. 1991. Expression of Epstein-Barr virus latent gene products in tumour cells of Hodgkin's disease. Lancet. 337:320-322.

35. Niedobitek, G., et al. 1992. Patterns of Epstein-Barr virus infection in non-neoplastic lymphoid tissue. Blood. 79:2520-2526.

36. Hargreaves, D.C., et al. 2001. A coordinated change in chemokine responsiveness guides plasma cell movements. J. Exp. Med. 194:45-56.

37. Mauri, C., Mars, L.T., and Londei, M. 2000. Therapeutic activity of agonistic monoclonal antibodies against CD40 in a chronic autoimmune inflammatory process. Nat. Med. 6:673-679. 